\title{
Pharmacoeconomic impact of use of the probiotic Lactobacillus reuteri DSM 17938 for prevention of necrotizing enterocolitis in extremely low-birth-weight infants
}

\author{
This article was published in the following Dove Press journal: \\ Research and Reports in Neonatology \\ 2 April 2013 \\ Number of times this article has been viewed
}

\author{
Mary Ann VT Dimaguila ${ }^{1,2}$ \\ Peter Gal 1,3,4 \\ Tiffany Wilson' \\ John E Wimmer Jr ${ }^{1,2}$ \\ McCrae Smith ${ }^{1,2}$ \\ Rita Q Carlos ${ }^{1,2}$ \\ Christie C Davanzo ${ }^{1,2}$ \\ J Laurence Ransom ${ }^{1,2}$ \\ 'Women's Hospital of Greensboro, \\ Cone Health, Greensboro, NC, USA; \\ ${ }^{2}$ Piedmont Neonatology, Greensboro, \\ NC, USA; ${ }^{3}$ Eshelman School of \\ Pharmacy, University of North \\ Carolina at Chapel Hill, Chapel Hill, \\ NC, USA; ${ }^{4}$ Greensboro Area Health \\ Education Center, Greensboro, \\ NC, USA
}

\begin{abstract}
Background: A recent study showed that use of Lactobacillus reuteri as probiotic prophylaxis decreased the necrotizing enterocolitis (NEC) rate from $15.1 \%$ to $2.5 \%$ in neonates with birth weight below $1000 \mathrm{~g}$. Given the controversies surrounding use of probiotics in neonatal intensive care units, we address one additional aspect of routine implementation of probiotics for NEC prophylaxis - the pharmacoeconomic impact.

Methods: Using data from our initial published experience, and continuing data collection after instituting a higher dose of $L$. reuteri, we measured the reduction in NEC in neonates with birth weight below $1000 \mathrm{~g}$. Cost savings from prior studies examining the cost and outcomes of medical and surgical NEC were used to calculate the financial impact of routine L. reuteri DSM 17938 prophylaxis.
\end{abstract}

Results: Medical records for 354 neonates were reviewed, 232 in the years before introduction of $L$. reuteri prophylaxis and 79 who received $L$. reuteri prophylaxis dosed at $0.1 \mathrm{~mL}$ daily and 43 neonates given a total daily dose of $0.2 \mathrm{~mL}$ as one or two doses. The incidence of NEC was significantly lower in the neonates who received L. reuteri (two of 122 neonates [1.6\%] versus 35 of 232 neonates [15.1\%]). The expected benefits for our neonatal intensive care unit per 100 extremely low-birth-weight neonates treated were four fewer deaths, five fewer cases of medical NEC, eight fewer cases of surgical NEC, one less patient with short-bowel syndrome, and a cost saving of approximately $\$ 2.2$ million.

Conclusion: Prophylactic initiation of $L$. reuteri as a probiotic for prevention of NEC in neonates with birth weight $\leq 1000 \mathrm{~g}$ is a cost-effective strategy during their stay in neonatal intensive care.

Keywords: necrotizing enterocolitis, probiotic, extremely low birth weight, Lactobacillus reuteri, pharmacoeconomics

\section{Background}

Probiotics have generally been shown to have favorable effects in preterm neonates, primarily reducing the rates of necrotizing enterocolitis (NEC). ${ }^{1-5}$ The rates of NEC, using different probiotic formulations, have been reduced by an average of $70 \%$, and mortality by $60 \%$, although the results may vary quite a bit, as noted by the wide confidence intervals noted in meta-analyses. ${ }^{6,7}$ Our recently published retrospective case series in neonates with birth weights of $\leq 1000 \mathrm{~g}$ documented an even larger reduction in NEC rates in this high-risk population. ${ }^{8} \mathrm{NEC}$ rates were reduced from $15.1 \%$ before routine Lactobacillus reuteri prophylaxis to $2.5 \%$ after routine prophylaxis
Correspondence: Peter Gal

Greensboro Area Health Education

Center, Suite 100, 200 East Northwood

Street, Greensboro, NC 2740I-1020,

USA

Tel +I 3368327932

Email peter.gal@conehealth.com 
with $L$. reuteri. Some authors caution against routine use of probiotics, citing concerns with product quality, confusion over the best strains of probiotic to select, and possible adverse effects. ${ }^{9,10}$ However, given the positive weight of evidence, it may be time to consider selected probiotic products for routine use in neonatal intensive care units (NICUs). To expand the debated issues further, we have performed a pharmacoeconomic analysis of our L. reuteri prophylaxis strategy utilizing the data from our recently published paper, ${ }^{8}$ and our additional experience with a higher-dose strategy.

\section{Methods}

\section{Data collection}

Data for this analysis include neonates administered $L$. reuteri DSM 17938 at a dose of $0.1 \mathrm{~mL}$ (approximately 550 million colony-forming units) daily, as in our original publication, ${ }^{8}$ and an additional 43 neonates administered a dose of $0.2 \mathrm{~mL}$ daily (110 million colony-forming units). This was a retrospective chart review of 354 consecutive patients comparing the rates of NEC in neonates with birth weight $\leq 1000 \mathrm{~g}$, before introduction of L. reuteri (January 2004-June 2009), with routine use of $L$. reuteri in neonates $\leq 1000 \mathrm{~g}$ birth weight (July 2009-June 2012), and was approved by the institutional review board and exempted from needing informed consent. Neonates with birth weight $\leq 1000 \mathrm{~g}$ were selected, since this is the highest at-risk group for NEC, ${ }^{11}$ and our NEC rate in this population was very high.

Medical records for all neonates in our NICU are routinely maintained in a unit-specific standardized database, NICU3, which was designed by one of the authors (MS). Patient data is also categorized for reporting our NICU outcomes as part of the Vermont Oxford reporting process. The hospital electronic patient record and paper-chart data were also used to collect study data. In addition to their demographic data, patients were classified as either medical NEC (Bell's stage IIA and IIB), or surgical NEC (Bell's stage IIIA and IIIB). Patients transferred to other neonatal units for surgical intervention had their status and outcome confirmed by the principal investigator (MAVTD). Patients who died from NEC in our study were all in the surgical NEC category.

\section{Pharmacoeconomic analysis}

Economic analysis was estimated based on prior analyses of the total cost for medical and surgical NEC. ${ }^{12,13}$ Based on the costs calculated in these studies, medical NEC costs above a parallel control group ranged from $\$ 73,700$ to $\$ 74,004$, and surgical NEC costs above controls ranged from
$\$ 186,200$ to $\$ 198,041$. These values were utilized for our cost analysis. We subtracted the approximate institutional cost of L. reuteri DSM 17938 (BioGaia, Stockholm, Sweden) for an average of 12 weeks of treatment for 100 patients with birth weight $\leq 1000 \mathrm{~g}$. We could not calculate actual costs for neonates with surgical NEC and some infants with medical NEC, because these babies are transferred to different, university-based, NICUs where surgery can be performed if necessary. Outcomes regarding confirmation of NEC at surgery for transferred infants were confirmed by the lead author. The cost of short bowel syndrome utilized the calculations of Spencer et $\mathrm{al}^{14}$ that average cost for the first year is $\$ 505,250$. The data from Cole et al ${ }^{15}$ suggest that one in four surgical NEC cases is likely to develop short-bowel syndrome. The cost numbers utilized in this analysis are similar to those used in the study of Bartick and Reinhold ${ }^{16}$ on the impact of suboptimal breastfeeding on costs.

Expected outcomes of NEC were extrapolated from the study by Cole et al, ${ }^{15}$ which reported on 5657 neonates with birth weight between 401 and $1000 \mathrm{~g}$. That study represented 16 NICUs involved in the National Institute of Child Health and Human Development neonatal research network, and thus represents a broad range of experiences. Cole et al ${ }^{15}$ identified 632 cases of NEC, $389(61.6 \%)$ with surgical and 243 (38.4\%) with medical NEC. Of the medical NEC cases, $61(15.7 \%)$ developed short-bowel syndrome. Mortality occurred in $49 \%$ of surgical NEC and $29 \%$ of medical NEC cases. We used this expected breakdown of outcomes for our expected NEC cases and pharmacoeconomic analysis. We also normalized our calculations per 100 patients to allow broader applicability in different NICU sizes.

\section{Results}

The rate of NEC in different treatment epochs on our NICU is illustrated in Figure 1. The difference in NEC rates for the control period and $L$. reuteri treatment period was $15.1 \%$ vs $1.6 \%$. The result is a number needed to treat of 7.4 to prevent one case of NEC. The NEC case breakdown for our 35 cases before using probiotic prophylaxis was 19 (54.3\%) surgical NEC and 16 (45.7\%) medical NEC. Our calculations for expected medical and surgical NEC events are extrapolated based on the report of Cole et $\mathrm{al}^{15}$ and illustrated in Figure 2. Their NEC case breakdown was $61.6 \%$ surgical NEC and $38.4 \%$ medical NEC. NEC cases in our NICU before implementation of routine $L$. reuteri prophylaxis would be expected to be 15 total cases, broken down as nine surgical and six medical. This compares favorably with the expected results from our actual percentage breakdown of eight 


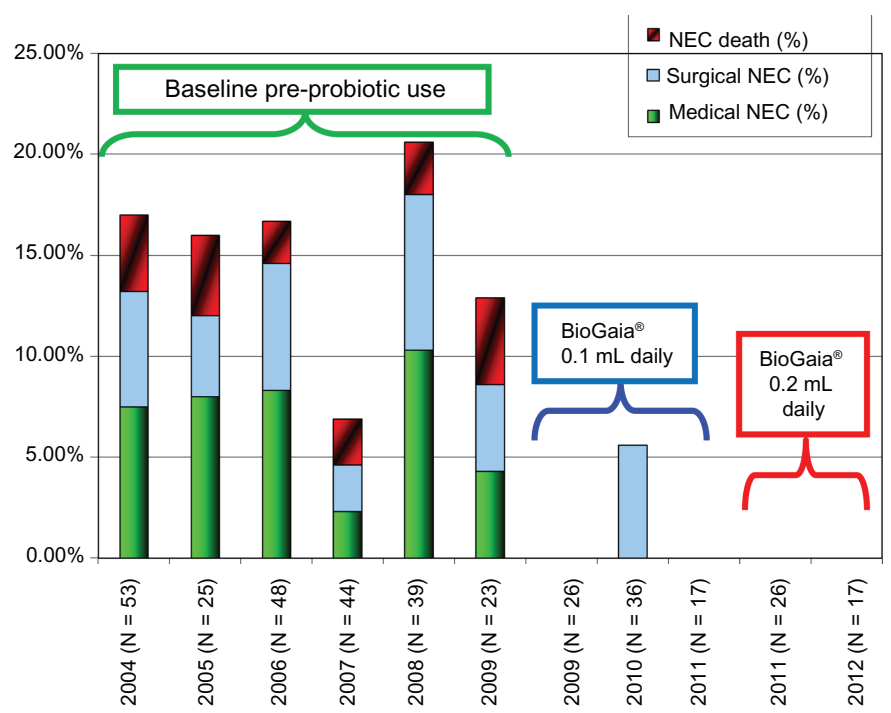

Figure I Annual rates of necrotizing enterocolitis (NEC) in neonates below $1000 \mathrm{~g}$ birth weight before and after initiation of Lactobacillus reuteri.

surgical and seven medical cases. Upon implementation of routine prophylaxis with $L$. reuteri, we calculate an expected one surgical and 0.6 medical NEC cases per 100 neonates with birth weight below $1000 \mathrm{~g}$ (Figure 2).

Assuming that 13 NEC cases can be avoided for every 100 neonates with birth weight below $1000 \mathrm{~g}$, a reasonable breakdown for NEC events avoided is five medical NEC, eight surgical NEC, and one case of short-bowel syndrome. Additionally, we can prevent four deaths due to NEC. The expected costs saved per 100 patients by implementing routine probiotic prophylaxis are: surgical NEC only $(\mathrm{n}=7), \$ 1,303,400-\$ 1,386,280$; surgical NEC with short-bowel syndrome $(\mathrm{n}=1), \$ 505,250$; and medical NEC $(\mathrm{n}=5), \$ 368,500-\$ 370,020$. If we subtract the hospital

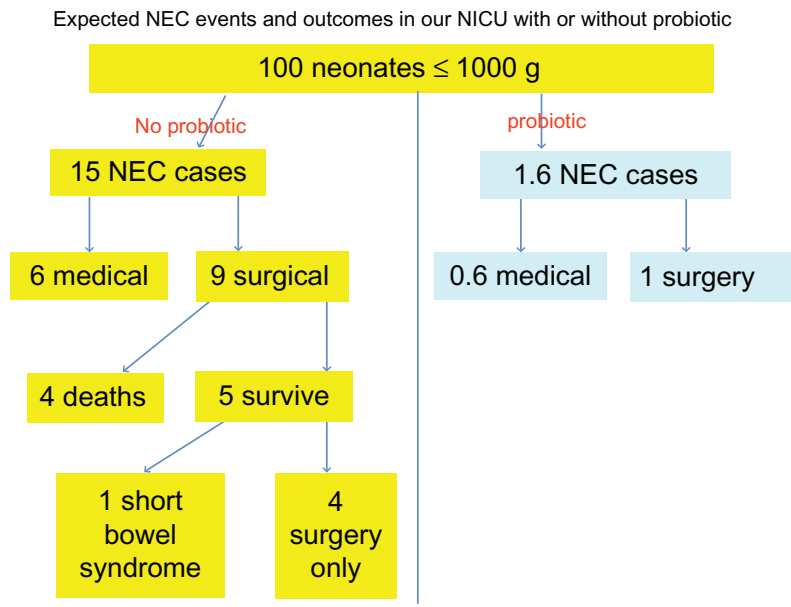

Figure 2 Expected necrotizing enterocolitis (NEC) events and outcomes for 100 neonates with birth weight $<1000 \mathrm{~g}$ admitted to our neonatal intensive care unit (NICU), before and after implementation of NEC prophylaxis with Lactobacillus reuteri. cost for L. reuteri DSM 17938 of approximately $\$ 3000$ for 100 patients, the total cost savings per 100 neonates given L. reuteri prophylaxis is $\$ 2,177,150-\$ 2,261,550$.

\section{Discussion}

The use of probiotics for NEC prophylaxis in preterm infants is controversial in the US, despite widespread use in many other countries. In a recent opinion piece, Janvier et al ${ }^{17}$ provide an elegant discussion of the difficulties of getting probiotics widely used in the US, and the reasons they really should be used, or at least parents should be offered a choice to use them. We would argue that the clinical benefits in preventing such a devastating disease as NEC, additional neonatal deaths, and the substantial cost savings noted in this subgroup justifies a policy of routine use at least in those neonates with birth weight $\leq 1000 \mathrm{~g}$. We cannot comment on which other probiotics would achieve benefits of a similar nature in the absence of head-to-head trials in extremely low-birth-weight infants. Clearly, numerous combinations of organisms, often with different strains of Lactobacillus or Bifidobacterium, have been tried in preterm infants below $1500 \mathrm{~g}$ birth weight with varying success. ${ }^{2}$ In a recent meta-analysis, ${ }^{2}$ NEC reduction appears comparable with either organism alone or in combination. Alternatively, the combination of Lactobacillus and Bifidobacterium lowered mortality more than either bacterium alone. Neither bacterium alone or in combination reduced sepsis rates. Combining all data for different strains of Lactobacillus or Bifidobacterium is also risky, as the different strains of each may provide different outcomes. Consequently, extrapolating our results beyond the specific organism in this study is problematic. 
The use of probiotics is not without some known risks and theoretical concerns. These cannot be accounted for in our economic analysis with the results of our study, but are worth mentioning. They are elegantly noted in two recent commentaries about probiotics. ${ }^{18,19}$ The risk of acute infection in neonates exposed to probiotics appears low, as we did not observe any patient with Lactobacillus infection; however, some case reports exist, and it is fair to say this risk is not nonexistent.

Also, the long-term impact of probiotics cannot be excluded. The changes in gastrointestinal microbiotica in newborns cannot be assumed not to alter risk of later diseases. It is also theoretically possible that the widespread use of probiotics in an NICU will alter the microbial ecology of the entire NICU for the worse and promote organisms that are more pathogenic or harder to treat. These factors are not measurable or economically quantifiable at this time.

Several limitations impact our calculations. We recognize that our NEC rate of $15.1 \%$ prior to L. reuteri prophylaxis was higher than the rates recorded in other reports, and a more realistic number in other NICUs might be around $10 \%{ }^{20}$ This would reduce the economic impact. We are unable to explain our relatively high NEC rate, since no obvious predisposing factors stood out. As noted in our previous study, ${ }^{8}$ variables thought to affect NEC rates were similar. We also could not consider the costs of known growth and neurodevelopmental consequences of NEC, ${ }^{21,22}$ which would have caused a significant underestimation of the pharmacoeconomic impact of prophylactic probiotics, not to mention a substantial personal toll on families. We have not achieved universal breastfeeding in our NICU at this time, although it is a recent goal. If we achieve this, it may also result in reduced NEC and would reduce the pharmacoeconomic impact of probiotics. However, in the study by Lin et $\mathrm{al}^{3}$, in infants below $1500 \mathrm{~g}$ birth weight, all were fed breast milk, NEC was cut from $6.5 \%$ to $1.8 \%$, and NEC + death reduced from $9.2 \%$ to $1.8 \%$. In the subgroup of patients with birth weight $500-1000 \mathrm{~g}$, NEC rates fell from $8.9 \%$ to $3.9 \%$, and NEC + death rates fell from $16.5 \%$ to $3.9 \%$. Thus, expectations of substantial benefits would remain even if breastfeeding is uniformly successful.

\section{Conclusion}

Prophylactic initiation of $L$. reuteri as a probiotic for prevention of NEC resulted in a statistically significant reduction in NEC. Our results support that in addition to the clinical benefits realized by initiating probiotics to prevent NEC, the short-term economic impact is large, saving approximately
$\$ 2$ million for every 100 neonates with birth weight $\leq 1000 \mathrm{~g}$ receiving L. reuteri DSM 17938.

\section{Authors' contributions}

The authors on this paper all participated in implementation of the L. reuteri prophylaxis protocol. The neonatologists were responsible for the final decision regarding NEC classification. All authors read and approved the final manuscript. The main database used to capture patient information was designed by MS. Data collection was primarily performed by TW in her role as Neonatal Pharmacotherapy Fellow at Women's Hospital, and monitored by the principal investigators MVD and PG. Pharmacoeconomic analysis was performed by $\mathrm{PG}$.

\section{Authors' information}

Drs Dimaguila, Wimmer, Ransom, Carlos, Smith, and Davanzo are neonatologists in Piedmont Neonatology PC and service the NICU at Women's Hospital of Greensboro as part of the Department of Neonatology. Dr Gal is a Neonatal Pharmacotherapy specialist who is a member of the Neonatology and Pharmacy Departments at Women's Hospital of Greensboro. He is also a Professor at University of North Carolina at Chapel Hill, Eshelman School of Pharmacy; and Director of Graduate Pharmacy Education, Greensboro Area Health Education Center, Greensboro, NC. Dr Wilson is a Neonatal Pharmacotherapy Fellow at Women's Hospital of Greensboro. Dr Wimmer is also Medical Director of the NICU at Women's Hospital of Greensboro. Dr Dimaguila directs the nutrition team and was responsible for implementation of probiotic prophylaxis as a standard of practice in the NICU.

\section{Disclosure}

The initial study was partially funded through a grant from BioGaia Inc. The authors have no further conflicts of interest to declare.

\section{References}

1. Awad H, Mokhtar H, Iman SS, Gad GI, Hafez H, Aboushady N. Comparison between killed and living probiotic usage versus placebo for the prevention of necrotizing enterocolitis and sepsis in neonates. Pak J Biol Sci. 2010;13:253-262.

2. Wang Q, Dong J, Zhu Y. Probiotic supplement reduces the risk of necrotizing enterocolitis and mortality in preterm very low-birth-weight infants: an updated meta-analysis of 20 randomized, controlled trials. J Pediatr Surg. 2012;47:241-248.

3. Lin $\mathrm{HC}, \mathrm{Hsu} \mathrm{CH}$, Chen $\mathrm{HL}$, et al. Oral probiotics prevent necrotizing enterocolitis in very low birth weight preterm infants: a multicenter, randomized, controlled trial. Pediatrics. 2008;122:693-700.

4. Lin $\mathrm{HC}, \mathrm{Su} \mathrm{BH}$, Chen AC, et al. Oral probiotics reduce the incidence and severity of necrotizing enterocolitis in very low birth weight infants. Pediatrics. 2005;115:1-4. 
5. Bin-Nun A, Bromiker R, Wilschanski M, et al. Oral probiotics prevent necrotizing enterocolitis in very low birth weight neonates. J Pediatr. 2005;147:192-196.

6. AlFaleh K, Anabrees J, Bassler D, Al-Kharfi T. Probiotics for prevention of necrotizing enterocolitis in preterm infants. Cochrane Database Syst Rev. 2011;3:CD005496.

7. Deshpande G, Rao S, Patole S, Bulsara M. Updated meta-analysis of probiotics for preventing necrotizing enterocolitis in preterm infants. Pediatrics. 2010;125:921-930.

8. Hunter C, Dimaguila MAVT, Gal P, et al. Effect of routine probiotic, Lactobacillus reuteri DSM 17938, use on rates of necrotizing enterocolitis in neonates with birthweight $<1000$ grams: a sequential analysis. BMC Pediatr. 2012;12:142.

9. Neu J. Routine probiotics for premature infants: let's be careful! J Pediatr. 2011;158:672-674.

10. Soll RF. Probiotics: are we ready for routine use? Pediatrics. 2010; 125:1071-1072.

11. Fitzgibbons SC, Ching Y, Yu D, et al. Mortality of necrotizing enterocolitis expressed by birth weight categories. J Pediatr Surg. 2009;44: 1072-1075.

12. Ganapathy V, Hay JW, Kim JH. Costs of necrotizing enterocolitis and cost-effectiveness of exclusively human milk-based products in feeding extremely premature infants. Breastfeed Med. 2012;7:29-37.

13. Bisquera JA, Cooper TR, Berseth CL. Impact of necrotizing enterocolitis on length of stay and hospital charges in very low birth weight infants. Pediatrics. 2002;109:423-428.

14. Spencer AU, Kovacevich D, McKinney-Barnett M, et al. Pediatric short-bowel syndrome: the cost of comprehensive care. Am J Clin Nutr. 2008;88:1552-1559.
15. Cole CR, Hansen NI, Higgins RD, Ziegler TR, Stoll BJ; Eunice Kennedy Shriver NICHD Neonatal Research Network. Very low birth weight preterm infants with surgical short bowel syndrome: incidence, morbidity and mortality, and growth outcomes at 18 to 22 months. Pediatrics. 2008;122:e573-e582.

16. Bartick M, Reinhold A. The burden of suboptimal breastfeeding in the United States: a pediatric cost analysis. Pediatrics. 2010;125: e1048-e1056.

17. Janvier A, Lantos J, Barrington $\mathrm{K}$. The politics of probiotics: probiotics, necrotizing enterocolitis and the ethics of neonatal research. Acta Paediatr. 2013;102:116-118.

18. Beattie LM, Hansen R, Barclay AR. Probiotics for preterm infants: confounding features warrant caution. Pediatrics. 2010;126 e742-e743.

19. Millar M, Wilks M, Fleming P, Costeloe K. Should the use of probiotics in the preterm be routine? Arch Dis Child Fetal Neonatal Ed. 2012;97: F70-F74.

20. Maheshwari A, Corbin LL, Schelonka RL. Neonatal necrotizing enterocolitis. Res Rep Neonatol. 2011;1:39-53.

21. Salhab WA, Perlman JM, Silver L, Broyles RS. Necrotizing enterocolitis and neurodevelopmental outcome in extremely low birth weight infants $<1000$ g. J Perinatol. 2004;24:534-540.

22. Pike K, Brocklehurst P, Jones D, et al. Outcomes at 7 years for babies who developed neonatal necrotising enterocolitis: the ORACLE Children Study. Arch Dis Child Fetal Neonatal Ed. 2012;97: F318-F322.
Research and Reports in Neonatology

\section{Publish your work in this journal}

Research and Reports in Neonatology is an international, peer-reviewed, open access journal publishing original research, reports, editorials, reviews and commentaries on neonatal health. The manuscript management system is completely online and includes a very quick and fair

\section{Dovepress}

peer-review system. Visit http://www.dovepress.com/testimonials.php to read real quotes from published authors. 